\title{
Factors Associated with Complicated Grief in Students Who Survived the Sewol Ferry Disaster in South Korea
}

\author{
So Hee Lee ${ }^{1}$, Hee Sun Nam², Hak Beom Kim³ ${ }^{3}$, Eun Ji Kim ${ }^{凶}$, Jin-Won Noh ${ }^{凶}$, and Jeong-Ho Chae ${ }^{5}$ \\ ${ }^{1}$ Department of Psychiatry, National Medical Center, Seoul, Republic of Korea \\ ${ }^{2}$ Maumtodoc Mental Health Clinic, Ansan, Republic of Korea \\ ${ }^{3}$ Children Welfare Center, Incheon, Republic of Korea \\ ${ }^{4}$ Department of Healthcare Management, Eulji University, Seongnam, Republic of Korea \\ ${ }^{5}$ Department of Psychiatry, Seoul St. Mary's Hospital, The Catholic University of Korea College of Medicine, Seoul, Republic of Korea
}

\begin{abstract}
Objective The Sewol ferry disaster caused shock and grief in South Korea. The aim of this study was to identify the factors associated with symptoms of complicated grief (CG) among the surviving students 20 months after that disaster.

Methods This study was conducted using a cross-sectional design and a sample of 57 students who survived the Sewol ferry disaster. Data were collected using the following instruments: Inventory of Complicated Grief (ICG), the Lifetime Incidence of Traumatic EventsChild, the Child Report of Post-Traumatic Symptoms (CROPS), KIDSCREEN-27, Family Adaptability and Cohesion Evaluation ScalesIII, the Peri-traumatic Dissociation-Post-traumatic Negative Beliefs-Post-traumatic Social Support scale, and the Strengths and Difficulties Questionnaire. A generalized linear model using a log link and Poisson distribution was performed to identify factors associated with symptoms of CG.
\end{abstract}

Results The mean score on the ICG was 15.57 (standard deviation: 12.72). Being born in 1999, a higher score on the CROPS and a lower score in autonomy and relationship with parents on the KIDSCREEN-27 were related to higher levels of CG.

Conclusion Twenty months after the Sewol ferry disaster, $24.5 \%$ of surviving students were suffering from CG. This study uncovered a vulnerable population of bereaved children at high risk for CG.

Psychiatry Investig 2018;15(3):254-260

Key Words Sewol ferry disaster, Surviving students, Complicated grief, Associated factors.

\section{INTRODUCTION}

On April 16, 2014, the Sewol ferry sank in the West Sea off South Korea. Only 181 of the 476 passengers survived. A total of 325 Danwon high school students were on a school trip, and 250 of them died. The Sewol ferry disaster caused negative emotional reactions in the public, including anger, sadness, and anxiety. ${ }^{1}$ The Sewol ferry disaster has been a big issue

Received: February 27, 2017 Revised: April 23, 2017

Accepted: May 4, 2017

$\square$ Correspondence: Eun Ji Kim

Maumtodoc Mental Health Clinic, 174 Gwangdeok-daero, Danwon-gu, Ansan 15470, Republic of Korea

Tel: +82-31-483-5989, Fax: +82-31-483-5987

E-mail: happymom.kim@gmail.com

$\triangle$ Correspondence: Jin-Won Noh, PhD

Department of Healthcare Management, Eulji University, 553 Sanseong-daero, Sujeong-gu, Seongnam 13135, Republic of Korea

Tel: +82-31-740-7235, Fax: +82-31-740-7172, E-mail: jinwon.noh@gmail.com (c) This is an Open Access article distributed under the terms of the Creative Commons Attribution Non-Commercial License (http://creativecommons.org/licenses/bync/4.0) which permits unrestricted non-commercial use, distribution, and reproduction in any medium, provided the original work is properly cited. with many claims made regarding the captain's and crewmembers' responsibility, the government's deficient response, and the slow response by rescue teams. According to one study that relied on the AcciMap methodology, the disaster was the result of a series of lapses and disregard for safety across different levels of government and regulatory bodies, the Chonghaejin ship company, and the Sewol's crewmembers. ${ }^{2}$

A study that evaluated the mental health effects of this disaster on the community of Ansan, where most victims and survivors resided, showed that residents there had a significantly higher prevalence of psychiatric disturbances than those in control communities ${ }^{3}$; the depression rate among the respondents from Ansan was 11.8\% and 18.4\% reported suicidal ideation. Descriptive disaster mental health studies have reported that many (11-38\%) distressed individuals presenting for evaluation at shelters and family assistance centers have stress-related and adjustment disorders, such as bereavement, major depression, and substance use disorders. ${ }^{4}$ When analyzing the impact of a major disaster on the mental 
health of a well-studied cohort, estimates of the exposure to the Canterbury earthquakes accounted for $10.8-13.3 \%$ of overall mental disorders in the 35 -year-old age cohort. ${ }^{5}$ While natural disasters are perceived as unavoidable and usually develop more slowly, human-caused disasters are assumed to be caused by neglect, carelessness, and failures in technology or malevolence. ${ }^{6}$ Hence, the latter have a more profound effect on victims than the former.

Students who survived the Sewol ferry disaster experienced not only a life-threatening traumatic event but also loss of their close friends. It is now generally agreed that grief refers to a wide variety of emotional, cognitive, and behavioral responses that normally follow the death of a loved one. ${ }^{7}$ For some, the grieving process may be distorted, resulting in what is now termed complicated grief (CG), a form of chronic grief with intense separation distress related to the loss. ${ }^{8}$

However, few studies are available on CG among disasterbereaved South Koreans after the ferry disaster. Bereaved people were at high risk for CG, which may have led to significant distress and impairment in their health. Understanding factors associated with CG is important to identify bereaved individuals who are at high risk and to provide targeted interventions and treatments.

The aim of this study was to identify factors associated with symptoms of CG among the surviving students 20 months after the Sewol ferry disaster. We hypothesized that the symptoms of CG among the surviving students would be severe and that demographic characteristics and psychological variables would be associated with symptoms of CG in this population. Thus, the aims of this study were to 1 ) investigate symptoms of CG in surviving students 20 months after the Sewol ferry disaster and 2) identify factors (demographic characteristics and psychological variables) associated with symptoms of CG.

\section{METHODS}

\section{Participants}

This study was performed with Danwon high school students who survived the Sewol ferry disaster in South Korea. We initially distributed questionnaires to the 75 students and obtained responses from 57 students, after excluding four with insufficient responses. This cross-sectional study was conducted in December 2015, 20 months after the Sewol ferry disaster.

\section{Variables and measurements}

Socioeconomic variables including sex, birth year, birth order position, household income, type of residence, and type of medical insurance were included. Participants also completed the Inventory of Complicated Grief (ICG), Lifetime Incidence of Traumatic Events-Child (LITE-C), Child Report of Post-traumatic Symptoms (CROPS), KIDSCREEN-27, Family Adaptability and Cohesion Evaluation Scales (FACES-III), Peri-traumatic Dissociation-Post-traumatic Negative Beliefs-Post-traumatic Social Support (PTD-PTNB-PTSS) scale, and the Strengths and Difficulties Questionnaire (SDQ).

Birth year was categorized as 1997, 1998, and 1999. Birth order position was classified as first born, second born, and third born, or younger. Household income was based on South Korean Won (KRW) per annum and classified into three categories $<30,000,000 \mathrm{KRW}$ (approximately 25,000 USD), $30,000,000-60,000,000 \mathrm{KRW}$, and $>60,000,000 \mathrm{KRW}$. Type of residence was categorized as self-occupied, deposit-basis rent, and monthly-basis rent. Type of medical security was classified as National Health Insurance, Medical Aid recipient, and the National Basic Livelihood Act (NBLA) recipient. The NBLA program was launched in October 2000 to support households with less than a designated minimum cost of living per month (approximately 1,500 USD for a household of four people as of 2016). The program is often considered a proxy measure of economic status in South Korea.

The dependent variable, ICG, is a self-report measure that assesses the severity of CG symptoms. ${ }^{9}$ Each of the 19 items is rated using a 5-point Likert scale ranging from 0 (not at all) to 4 (severe). Internal consistency is high (Cronbach's $\alpha=0.94$ ) and its concurrent validity in relation to the Beck Depression Inventory is acceptable $(\mathrm{r}=0.67, \mathrm{p}<0.001) .{ }^{10} \mathrm{~A}$ score of 25 is regarded as the cutoff to distinguish whether a bereaved subject has the symptoms of CG. ${ }^{10}$

The LITE-C is a checklist assessing trauma or losses that children have experienced; it also assesses the age at which the trauma occurred, how many times it occurred, and how bad the child felt at the time. The items on the LITE-C include car accidents, house fires, death of a family member, exposure to threats, sexual assaults, witness to violence, and other potentially upsetting events. The test-retest reliability of the LITE has an $r$-value of $0.80(p<0.001)$. The child completes the LITE-C measure on their own, and a clinician reviews it to rate the entire set of responses as 1 (no trauma or loss), 2 (possible trauma/loss), 3 (probable trauma/loss), or 4 (trauma/ loss)..$^{10}$

CROPS $^{11}$ is a screening tool for post-traumatic symptoms in children who may or may not have an identified childhood traumatic event. The CROPS comprises 26 items assessing post-traumatic symptoms over the previous 7 days. All the items are rated on a 3-point Likert scale ( $0=$ "none," $1=$ "some," $2=$ "a lot"). The total score is calculated by adding all item responses, with higher scores indicating more post-traumatic symptoms. Cutoff identify what constitutes a clinical concern; at 
present, the best cutoff for CROPS scores is $18 .{ }^{11}$ The CROPS was translated into Korean by Lee et al., ${ }^{12}$ who also confirmed its reliability and validity.

The KIDSCREEN (KIDSCREEN-52) instrument assesses the subjective health and wellbeing (health-related quality of life) of children and adolescents. The tool was developed as a self-report measure applicable for healthy and chronically ill children and adolescents aged 8-18 years. The KIDSCREEN-27 was developed as a shorter version of the KIDSCREEN-52 with minimum information loss and good psychometric properties. The resulting KIDSCREEN-27 has five dimensions. All five dimensions are Rasch scales: Physical Wellbeing (five items), Psychological Wellbeing (seven items), Autonomy \& Parents (seven items), Peers \& Social Support (four items), and School Environment (four items). ${ }^{13}$

FACES III was developed to assess two major dimensions on the circumflex model: adaptation and cohesion of the family. The measure can be used with families across the life-cycle from newlyweds with no children to retired couples. The authors report that children $>12$ years old can complete the FACES III to allow for comparisons between family members. Family cohesion assesses the degree of separation or connection of family members to the family. There are four levels of family cohesion ranging from extreme low cohesion to extreme high cohesion: disengaged, separated, connected, and enmeshed. Similarly, there are four levels of adaptability: rigid, structured, flexible, and chaotic. Family members answer 20 statements (e.g., family members ask each other for help; it is hard to identify the leaders in our family) by estimating the relative truth of the statement. A second scale measures what the family member would like in an ideal situation (e.g., family members would ask each other for help; we would know who the leader is in our family). ${ }^{14}$

The SDQ is a brief behavioral screening questionnaire for 3-16-year-olds. The SDQ asks about 25 attributes, some positive and others negative. It exists in several versions to meet the needs of researchers, clinicians, and educators. The 25 items are divided among 5 scales: $1=$ emotional symptoms; $2=$ conduct problems; $3=$ hyperactivity/inattention; $4=$ peer relationship problems; $5=$ prosocial behavior; dimensions 1 to 4 can be added together to generate a total difficulties score. This self-report version is suitable for young people aged 11-16 years, depending on their level of understanding and literacy.

The PTD-PTNB-PTSS is a shortened version of the Posttrauma Risk checklist (PRC), ${ }^{12}$ which is a useful and validated Korean instrument to evaluate risk factors for post-traumatic stress disorder (PTSD). The PRC consists of 57 items and measures PTD, PTNB, and PTSS. The PTD-PTNB-PTSS is made up of eight items selected from the PRC (three PTD items, 10 PTNB items, and five PTSS items) that have been changed from yes or no answers to a 5-point Likert scale.

\section{Ethical statement}

The study was approved by the Ethics Committee of the National Medical Center (IRB No. H-1505-054-002). Written informed consent was obtained from all participants. All participants were informed that they could withdraw from the study at any time.

\section{Statistical analysis}

Descriptive analyses of the data were performed to examine the subjects' general characteristics, and frequencies, percentages, means, and standard deviations were calculated. The dependent variable, ICG score, had a marked positive skew, which violated the distributional assumption of normality, making a normal regression inappropriate. Therefore, we assumed that distress had a Poisson distribution, such that the conditional mean was roughly equivalent to the variance. A generalized linear model using a log link and Poisson distribution was performed. ${ }^{17}$ No evidence of significant multicollinearity was found among the variables when examining variance inflation factors. Stata/MP 14.2 software (StataCorp, College Station, TX, USA) was used for all statistical analyses, and the threshold for significance test was $\mathrm{p}<0.05$ (two sided).

\section{RESULTS}

Result of sociodemographic and health-related characteristics of the study participants are summarized in Table 1. Of the 57 participants included in our study, 29 were males and 28 were females. More than three-quarters (82.46\%) of the participants were born in 1997; and 36.84\% were first born, $47.37 \%$ were second born, and $7.02 \%$ were third born or younger. About $25 \%$ of participants reported incomes $<30,000,000$ KRW, 22.81\% reported 30,000,000-60,000,000 KRW, and $10.53 \%$ reported $>60,000,000 \mathrm{KRW}$ per annum. For type of residence, $47.37 \%$ of participants reported living in a self-occupied house, $28.07 \%$ reported living in a deposit-basis rental house, and $7.02 \%$ reported living in a monthly-basis rental house. For type of medical insurance, $38.60 \%$ of respondents received National Health Insurance, 3.51\% were Medical Aid recipients, and 5.26\% were NBLA recipients.

The results of the clinical classification of the study population are summarized in Table 2. About 16\% (Autonomy \& Parents dimension), 43.83\% (Psychological Wellbeing, Peers \& Social Support, and Autonomy \& Parents dimension), and 21.03\% (School Environment Dimension) of the participants were classified in the clinical group according to the KIDSCREEN-27. About 12\% (prosocial behavior dimension), 
5.26\% (emotional symptoms dimension), 14.03\% (conduct problems dimension), $8.77 \%$ (hyperactivity/inattention dimension), and $8.77 \%$ (peer relationship problems dimension) of participants were classified as the clinical group by the SDQ. About 25\% and 26.29\% of participants were classified in the clinical group by ICG and CROPS scores, respectively.

Table 1. General characteristics of the study population

\begin{tabular}{|c|c|c|c|}
\hline Variable & Subcategory & $\mathrm{N}(\%)$ & $\mathrm{M}(\mathrm{SD})$ \\
\hline \multirow[t]{2}{*}{ Sex } & Male & $29(50.88)$ & \\
\hline & Female & $28(49.12)$ & \\
\hline \multirow[t]{3}{*}{ Birth year } & 1998 & $9(15.79)$ & \\
\hline & 1997 & $47(82.46)$ & \\
\hline & 1999 & $1(1.75)$ & \\
\hline \multirow[t]{4}{*}{ Birth order position } & First & $21(36.84)$ & \\
\hline & Second & $27(47.37)$ & \\
\hline & Third or younger & $4(7.02)$ & \\
\hline & Not answered & $5(8.77)$ & \\
\hline \multirow{4}{*}{$\begin{array}{l}\text { Household income } \\
\text { (ten thousand KRW) }\end{array}$} & Less than 3,000 & $14(24.56)$ & \\
\hline & 3,000 to 6,000 & $13(22.81)$ & \\
\hline & More than 6,000 & $6(10.53)$ & \\
\hline & Not answered & $24(42.11)$ & \\
\hline \multirow[t]{4}{*}{ Type of residence } & Self-occupied & $27(47.37)$ & \\
\hline & Deposit basis rent & $16(28.07)$ & \\
\hline & Monthly basis rent & $4(7.02)$ & \\
\hline & Not answered & $10(17.54)$ & \\
\hline \multirow[t]{4}{*}{ Type of medical insurance } & National health insurance & $21(36.84)$ & \\
\hline & Medical aid recipients & $2(3.51)$ & \\
\hline & NBLA recipients & $3(5.26)$ & \\
\hline & Not answered & $31(54.39)$ & \\
\hline LITE-C & $(\min =0, \max =8)$ & & $3.14(1.98)$ \\
\hline \multirow[t]{6}{*}{ KIDSCREEN-27 } & Total score $(\min =64, \max =135)$ & & $93.36(19.48)$ \\
\hline & Physical well-being $(\min =8, \max =25)$ & & $16.21(4.19)$ \\
\hline & Psychological well-being $(\min =12, \max =35)$ & & $24.91(5.56)$ \\
\hline & Peers $\&$ social support $(\min =6, \max =20)$ & & $14.12(3.36)$ \\
\hline & Autonomy \& parents $(\min =14, \max =35)$ & & $23.91(5.82)$ \\
\hline & School environment $(\min =4, \max =20)$ & & $12.88(3.89)$ \\
\hline \multirow[t]{3}{*}{ FACES } & Total score $(\min =35, \max =100)$ & & $67.98(15.88)$ \\
\hline & Cohesion $(\min =10, \max =50)$ & & $33.37(8.61)$ \\
\hline & Adaptability $(\min =10, \max =50)$ & & $33.14(8.30)$ \\
\hline \multirow[t]{4}{*}{ PTD-PTNB-PTSS } & Total score $(\min =15, \max =56)$ & & $22.96(8.99)$ \\
\hline & Peritraumatic dissociation $(\min =3, \max =15)$ & & $5.51(2.93)$ \\
\hline & Post-traumatic negative beliefs $(\min =10, \max =33)$ & & $16.54(6.34)$ \\
\hline & Post-traumatic social support $(\min =5, \max =15)$ & & $6.18(2.31)$ \\
\hline SDQ (difficulties) & $(\min =1, \max =27)$ & & $11.27(5.92)$ \\
\hline SDQ (strengths) & $(\min =3, \max =10)$ & & $6.64(1.93)$ \\
\hline ICG & $(\min =0, \max =47)$ & & $15.57(12.72)$ \\
\hline CROPS & $(\min =0, \max =37)$ & & $12.51(9.49)$ \\
\hline
\end{tabular}

LITE-C: Lifetime Incidence of Traumatic Events for Student, FACES: Family Adaptability and Cohesion Evaluation Scales, PTD-PTNBPTSS: Peritraumatic dissociation-Post-Traumatic Negative Beliefs-Post-Traumatic Social Support, SDQ: Strength and Difficulties Questionnaire, ICG: Inventory of Complicated Grief, CROPS: Child Report of Post-traumatic Symptoms 
Table 3 summarizes the result from the generalized linear model identifying factors related to CG after controlling for sociodemographic and psychological factors. After adjusting for sex and birth year covariates, CG was significantly associated with birth year, the Autonomy \& Parents dimension of
KIDSCREEN-27, and CROPS scores. Respondents who were born in 1999 had significantly higher ICG scores compared to those who were born in 1998 [coef. $=0.884$, $\mathrm{p}=0.007,95 \%$ confidence interval (CI), 0.244-1.524]. Higher scores on the CROPS (coef. $=0.030, \mathrm{p}=0.017,95 \% \mathrm{CI}, 0.005-0.054$ ) were sig-

Table 2. Clinical classification of study population

\begin{tabular}{llcc}
\hline Variable & \multicolumn{1}{c}{ Subcategory } & Non-clinical group, N (\%) & Clinical group, N (\%) \\
\hline KIDSCREEN-27 & Physical well-being & $48(84.21)$ & $9(15.79)$ \\
& Psychological well-being & $32(56.14)$ & $25(43.86)$ \\
& Peers \& social support & $32(56.14)$ & $25(43.86)$ \\
& Autonomy \& parents & $32(56.14)$ & $25(43.86)$ \\
& School environment & $45(78.95)$ & $7(12.28)$ \\
SDQ & Prosocial behavior & $50(87.72)$ & $3(5.26)$ \\
& Emotional symptoms & $54(94.74)$ & $8(14.04)$ \\
& Conduct problems & $49(85.96)$ & $5(8.77)$ \\
ICG & Hyperactivity/inattention & $52(91.23)$ & $5(8.77)$ \\
CROPS & Peer relationship problems & $52(91.23)$ & $14(24.56)$ \\
\hline
\end{tabular}

SDQ: Strength and Difficulties Questionnaire, ICG: Inventory of Complicated Grief, CROPS: Child Report of Post-traumatic Symptoms

Table 3. Result from generalized linear models evaluating the impact of quality of life in the area of autonomy \& parental relationship, posttraumatic negative beliefs and difficulties on ICG

\begin{tabular}{|c|c|c|c|c|c|}
\hline Variables & Subcategory & Coef. & Robust SE & $\mathrm{p}$ & $95 \% \mathrm{CI}$ \\
\hline \multirow[t]{2}{*}{ Sex } & M & 0.000 & (reference) & & \\
\hline & $\mathrm{F}$ & 0.021 & 0.185 & 0.910 & $-0.342-0.384$ \\
\hline \multirow[t]{3}{*}{ Birth year } & 1998 & 0.000 & (reference) & & \\
\hline & 1997 & 0.096 & 0.237 & 0.686 & $-0.369-0.561$ \\
\hline & 1999 & 0.884 & 0.327 & 0.007 & $0.244-1.524$ \\
\hline LITE-C & & 0.007 & 0.026 & 0.790 & $-0.043-0.057$ \\
\hline \multirow[t]{5}{*}{ KIDSCREEN-27 } & Physical well-being & 0.015 & 0.037 & 0.692 & $-0.058-0.088$ \\
\hline & Psychological well-being & -0.003 & 0.022 & 0.883 & $-0.046-0.039$ \\
\hline & Peers \& social support & 0.019 & 0.042 & 0.647 & $-0.063-0.102$ \\
\hline & Autonomy \& parents & -0.051 & 0.024 & 0.032 & $-0.098--0.004$ \\
\hline & School environment & 0.011 & 0.028 & 0.702 & $-0.044-0.065$ \\
\hline \multirow[t]{2}{*}{ FACES } & Cohesion & -0.013 & 0.033 & 0.696 & $-0.077-0.051$ \\
\hline & Adaptability & 0.020 & 0.029 & 0.488 & $-0.037-0.078$ \\
\hline \multirow[t]{3}{*}{ PTD-PTNB-PTSS } & Peritraumatic dissociation & 0.000 & 0.040 & 0.998 & $-0.078-0.078$ \\
\hline & Post-traumatic negative beliefs & 0.030 & 0.017 & 0.074 & $-0.003-0.063$ \\
\hline & Post-traumatic social support & -0.003 & 0.057 & 0.964 & $-0.114-0.109$ \\
\hline \multirow[t]{2}{*}{ SDQ } & Difficulties & 0.017 & 0.016 & 0.295 & $-0.015-0.049$ \\
\hline & Strengths & 0.125 & 0.079 & 0.113 & $-0.030-0.279$ \\
\hline \multicolumn{2}{|l|}{ CROPS } & 0.030 & 0.013 & 0.017 & $0.005-0.054$ \\
\hline \multicolumn{6}{|c|}{$\mathrm{AIC}=9.759 ; \mathrm{BIC}=128.680$} \\
\hline
\end{tabular}

AIC: akaike information criterion, BIC: bayesian information criterion, LITE-C: Lifetime Incidence of Traumatic Events for Student, FACES: Family Adaptability and Cohesion Evaluation Scales, PTD-PTNB-PTSS: Peritraumatic dissociation-Post-Traumatic Negative Beliefs-PostTraumatic Social Support, SDQ: Strength and Difficulties Questionnaire, CROPS: Child Report of Post-traumatic Symptoms 
nificantly associated with higher ICG scores. On the other hand, higher scores on the Autonomy \& Parents dimension of KIDSCREEN-27 were significantly associated with lower ICG scores (coef. $=-0.051, \mathrm{p}=0.032,95 \% \mathrm{CI},-0.098$ to -0.004 ).

\section{DISCUSSION}

About $24.5 \%$ of surviving children were suggested to be suffering from CG 20 months after the Sewol ferry disaster. This study uncovered a vulnerable population of bereaved individuals at high risk for CG.

The percentage of CG cases in this study was higher than that in a general population-based bereavement study. A prevalence study of CG in a representative population-based sample reported that the conditional likelihood of developing CG after major bereavement was 6.7\%; the prevalence of CG in the general sample was $3.7 \%{ }^{18}$ Mental health disorders are more likely to be elevated after sudden and violent losses than after losses following natural deaths or illnesses, and the trajectory of recovery seems to be slower. The participants in this study experienced traumatic loss by a ferry accident. Additionally, deaths of the majority of the second grade, resentment of survivors, suicide of the vice-principal, and media effects disrupted normal functions at their school for a moderate period.

Second, the percentage of CG among participants of this study was lower than that in some studies of disaster-bereaved individuals. For instance, the percentage of CG among disaster-bereaved individuals was $47.7 \%$ at 2 years after the 2004 South-East Asian tsunami. ${ }^{19}$ Approximately $79 \%$ of the bereaved individuals had symptoms of CG 18 months after the Wenchuan earthquake. ${ }^{20}$ The participants in this study lost their friends, not their family members. Victims who lost a child had a higher level of CG compared with other types of loss in population-base bereavement study ${ }^{18}$ In addition, the participants in this study received appropriate psychiatric interventions by a school psychiatrist after the accident. Treatment interventions can effectively diminish CG symptoms. ${ }^{21}$ Receiving psychological counseling is associated with a lower level of CG. ${ }^{20}$

Third, 26.29\% of the participants suffered from PTSD after the tragic disaster, and the severity of PTSD was correlated with the level of CG. Higher scores on the CROPS were significantly associated with higher ICG scores. Raphael ${ }^{22}$ described bereavement and stress responses as two sets of phenomena that differ in important ways. Loss of a loved one or bereavement is associated with grief reactions and a traumatic event involving a personal life threat and/or witnessing another's gruesome death can be followed by a post-traumatic stress reaction. When death occurs under traumatic or vi- olent circumstances, also termed traumatic bereavement, a combination of grief and stress reaction or PTSD may occur. Traumatic grief has been used to denote a condition when PTSD symptoms impinge on grieving, such as when thoughts or reminders of the circumstances of death and the way the person died hinders positive reminiscing about the deceased. ${ }^{23}$

Fourth, note that nearly half of the participants reported a low quality of life (lower than the 16th percentile), especially in the dimensions of Psychological Wellbeing, Peers \& Social Support, and Autonomy \& Parents. This finding is explained by a previous study in which children who were exposed to trauma reported a poorer quality of life than non-exposed children. ${ }^{24}$ In addition, lower scores on the Autonomy \& Parents section of KIDSCREEN-27 were significantly associated with higher ICG scores. Autonomy \& Parents (seven items) examines the quality of child/adolescent and parent/care giver interactions and the extent to which the child/adolescent feels loved and supported by the family. Furthermore, it explores the level of autonomy that the child/adolescent perceives her/himself to possess, and the quality of financial resources perceived as available by the child/adolescent. We should note that both the government and the parents requested that the students be grouped together at a single hospital capable of appropriate psychiatric care, because they were young, emotionally susceptible adolescents. ${ }^{25}$ Our results support previous research emphasizing the importance of parental support for reducing psychological adversities among children after disasters. Although many adolescents exposed to earthquakes recover over time, some exhibit chronic, delayed-onset PTSD and depression, particularly those with poor relationships with their parents or those living in precarious economic conditions. ${ }^{26}$ Social support from parents or a classmate/friend was a significant protective factor associated with a lower likelihood of falling into a symptomatic pattern among children after Hurricane Katrina. ${ }^{27}$

Lastly, the SDQ classified from 5.26 to $14.03 \%$ of the participants into various clinical groups: 5.26\%, emotional symptoms; $8.77 \%$, hyperactivity/inattention; $8.77 \%$, peer relationship problems; $12 \%$, prosocial behavior; and $14.03 \%$, conduct problems. This finding was similar to the $13.6 \%$ prevalence of the SDQ clinical range for all difficulties among children who survived the 2011 Great East Japan Earthquake. ${ }^{28}$

The results of the present study were limited by some factors. First, the cross-sectional design makes it difficult to identify causal relationship among factors. As a result, a longitudinal study is recommended. Second, some sampling bias may have occurred, as only $75 \%$ of the surviving students participated in the study; therefore, the results should be interpreted with caution. Third, although the demographic characteristics and psychological variables were accounted for in the analysis, 
some additional factors, such as social support, self-efficacy, and coping style should be included in future studies.

In conclusion, despite these limitations, our study has provided multiple insights. This study identified factors associated with symptoms of CG among surviving students after the Sewol ferry accident. The results have unique implications for relief work with the bereaved population of the Sewol ferry accident and other disasters. According to our findings, the symptoms of CG among the surviving students were higher 20 months after Sewol ferry disaster than in a study of the general bereaved population. Being an older student, having more PTSD symptoms, and having a lower quality of life in the dimension of autonomy and parent relations were associated with symptoms of CG. The results of the study also inform strategies suitable for bereaved individuals after the Sewol ferry accident, including early assessment for those at high risk for CG, targeted intervention programs for the survivors with CG or PTSD, and education for the survivors' parents.

\section{Acknowledgements}

This study was supported by a grant from the Korean Mental Health Technology R\&D Project, Ministry of Health \& Welfare, Republic of Korea (HM15C1054).

\section{REFERENCES}

1. Woo H, Cho Y, Shim E, Lee K, Song G. Public trauma after the Sewol Ferry disaster: the role of social media in understanding the public Mood. Int J Environ Res Public Health 2015;12:10974-10983.

2. Lee S, Moh YB, Tabibzadeh M, Meshkati N. Applying the AcciMap methodology to investigate the tragic Sewol Ferry accident in South Korea. Appl Ergon 2017;59:517-525.

3. Yang HJ, Cheong HK, Choi BY, Shin MH, Yim HW, Kim DH, et al. Community mental health status six months after the Sewol ferry disaster in Ansan, Korea. Epidemiol Health 2015;37:e2015046.

4. North CS, Pfefferbaum B. Mental health response to community disasters: a systematic review. JAMA 2013;310:507-518.

5. Fergusson DM, Horwood LJ, Boden JM, Mulder RT. Impact of a major disaster on the mental health of a well-studied cohort. JAMA Psychiatry 2014;71:1025-1031.

6. Norris FH, Friedman MJ, Watson PJ. 60,000 disaster victims speak: Part II. Summary and implications of the disaster mental health research. Psychiatry 2002;65:240-260.

7. Stroebe MS, Folkman S, Hansson RO, Schut H. The prediction of bereavement outcome. Soc Sci Med 2006;63:2440-2451.

8. Prigerson HG, Vanderwerker LC, Maciejewski PK. A case for Inclusion of Prolonged Grief Disorder in DSM-V. In: Stroebe MS, Hansson R, Schut H, Stroebe W, Editors. Handbook of Bereavement Research and Practice. Washington, DC: American Psychological Association, 2008, p.165-186.

9. Shear K, Frank E, Houck PR, Reynolds CF 3rd. Treatment of complicated grief: a randomized controlled trial. JAMA 2005;293:2601-2608.
10. Prigerson HG, Maciejewski PK, Reynolds CF 3rd, Bierhals AJ, Newsom JT, Fasiczka A, et al. Inventory of complicated grief: a scale to measure maladaptive symptoms of loss. Psychiatry Res 1995;59:65-79.

11. Greenwald R, Rubin A. Assessment of posttraumatic symptoms in children: development and preliminary validation of parent and child scales. Res Soc Work Pract 1999;9:61-75.

12. Lee KM, Jeong SH, Lee WK, Chung US. Reliability and validity of the Korean version of the child report of post-traumatic symptoms (CROPS) and the parent report of post-traumatic symptoms (PROPS). J Korean Acad Child Adolesc Psychiatry 2011;22:169-181.

13. Ravens-Sieberer U, Auquier P, Erhart M, Gosch A, Rajmil L, Bruil J, et al. The KIDSCREEN-27 quality of life measure for children and adolescents: psychometric results from a cross-cultural survey in 13 European countries. Qual Life Res 2007;16:1347-1356.

14. Olson DH. FACES III (Family Adaptation and Cohesion Scales). St. Paul, MN: University of Minnesota; 1985.

15. Goodman R, Meltzer H, Bailey V. The strengths and difficulties questionnaire: a pilot study on the validity of the self-report version. Eur Child Adolesc Psychiatry 1998;7:125-130.

16. Joo H, Ahn H. Development and posttrauma risk checklist. Korean J Psychol Gen 2008;27:235-257.

17. Liang $\mathrm{K}$, Zeger S. Longitudinal data analysis using generalized linear models. Biometrika 1986;73:13-22.

18. Kersting A, Brähler E, Glaesmer H, Wagner B. Prevalence of complicated grief in a representative population-based sample. J Affect Disord 2011;131:339-343.

19. Kristensen P, Weisaeth L, Heir T. Predictors of complicated grief after a natural disaster: a population study two years after the 2004 SouthEast Asian tsunami. Death Stud 2010;34:137-150.

20. Hu XL, Li XL, Dou XM, Li R. Factors related to complicated grief among bereaved individuals after the Wenchuan earthquake in China. Chin Med J (Engl) 2015;128:1438-1443.

21. Wittouck C, Van Autreve S, De Jaegere E, Portzky G, van Heeringen K. The prevention and treatment of complicated grief: a meta-analysis. Clin Psychol Rev 2011;31:69-78.

22. Raphael B. Preventive intervention with the recently bereaved. Arch Gen Psychiatry 1977;34:1450-1454.

23. Cohen JA, Mannarino AP, Greenberg T, Padlo S, Shiple C. Childhood traumatic grief. Trauma Viol Abuse 2002;3:307-327.

24. Alisic E, van der Schoot TA, van Ginkel JR, Kleber RJ. Looking beyond posttraumatic stress disorder in children: posttraumatic stress reactions, posttraumatic growth, and quality of life in a general population sample. J Clin Psychiatry 2008;69:1455-1461.

25. Park JH, Cho H, Kim JY, Song JH, Moon S, Cha SH, et al. The Sewol Ferry disaster: experiences of a community-based hospital in Ansan city. Disaster Med Public Health Prep 2017;11:389-393.

26. Tang W, Zhao J, Lu Y, Yan T, Wang L, Zhang J, et al. Mental health problems among children and adolescents experiencing two major earthquakes in remote mountainous regions: a longitudinal study. Compr Psychiatry 2017;72:66-73.

27. Lai BS, Kelley ML, Harrison KM, Thompson JE, Self-Brown S. Posttraumatic stress, anxiety, and depression symptoms among children after hurricane Katrina: a latent profile analysis. J Child Fam Stud 2015;24: 1262-1270.

28. Kikuya M, Miyashita M, Yamanaka C, Ishikuro M, Sato Y, Obara T, et al. Protocol and research perspectives of the ToMMo child health study after the 2011 great east Japan earthquake. Tohoku J Exp Med 2015;236:123-130. 